Objectives: To evaluate outcomes of rapid dose escalation regimen of MTX compared with conventional treatment.

Methods: We implemented a randomized, controlled trial that enrolled patients with RA who fulfilled all of the following criteria: 20 to 70 years-old, disease duration $\leq 2$ years, SDAI $\geq 11$, and without prior use of MTX, tacrolimus (TAC) or biologics. Patients were randomized into rapid escalation (RE) group or conventional treatment (CT) group at 1:1 ratio. In RE group, doses of MTX were escalated up to $0.25 \mathrm{mg} / \mathrm{kg} / \mathrm{wk}$ within 8 wks after start of MTX and increased maximum tolerable dose or $16 \mathrm{mg} / \mathrm{wk}$ until wk 12 . If a patient achieved SDAI remission at wk 12, MTX was continued at the same dose. If a patient did not achieve SDAI remission at wk 12 or showed intolerance to MTX, use of TAC or TNF inhibitor (TNFi) were allowed. In CT group, patients were treated with either MTX, TAC, salazosulfapyridine, or bucillamine by the discretion of physicians until wk 12. If a patient achieved SDAl remission at wk 12, same treatment was continued. If a patient did not achieve remission at wk 12, use of TNFi was allowed. Patients were treated by the discretion of physicians at wk 24 and onward. We set two primary endpoints; the percentage of patients achieving SDAI remission and Boolean remission at wk 24. We planned to enroll 120 patients per arm based on expected SDAI remission rates at wk 24 , alpha and beta errors and dropout rates.

Results: Enrollment was terminated prematurely and all patients were followed for 48 wks. Of 115 enrolled patients, 57 were randomly assigned to RE group and 58 to CT group. Baseline demographics were similar between the two groups. The median baseline values (RE vs. CT) were 24.9 and 25.9 for SDAI, 0.88 and 0.69 for $\mathrm{HAQ}$, and 0.64 and 0.61 for EQ-5D, respectively. At wk 24, the percentages of patients achieving remission in RE and CT groups were $42 \%$ and $28 \%$ by SDAI criteria $\left(p=0.1, x^{2}\right.$ test), and $35 \%$ and $17 \%\left(p=0.03, x^{2}\right.$ test) by Boolean criteria, respectively. Median values of $H A Q$ at wk 24 in RE and CT groups were 0 and 0.13 ( $p=0.096, M-W U$ test), and those of EQ-5D were 0.78 and $0.77(p=0.12$, $\mathrm{M}-\mathrm{W} \cup$ test), respectively. At wk 48 , these values were not statistically different between the two groups. There were no significant differences between the two groups with incidence of severe adverse events.

Conclusions: The rapid dose escalation regimen of MTX provided significantly superior Boolean remission rate and tended to provide superior SDAI remission than conventional treatment in patients with early RA in the short term.

Disclosure of Interest: M. Tsutsumino Grant/research support from: Ayumi Pharmaceutical Co., Chugai Pharmaceutical Co., Ltd., Eisai Co., Ltd., Nippon Kayaku Co., Ltd., Taisho Toyama Pharmaceutical Co., Ltd., Takeda Pharmaceutical Co., Ltd., Mitsubishi Tanabe Pharma Co., and Teijin Pharma Ltd., R. Sakai Grant/research support from: Ayumi Pharmaceutical Co., Chugai Pharmaceutical Co., Ltd., Eisai Co., Ltd., Nippon Kayaku Co., Ltd., Taisho Toyama Pharmaceutical Co., Ltd., Takeda Pharmaceutical Co., Ltd., Mitsubishi Tanabe Pharma Co., Teijin Pharma Ltd., and Bristol-Meyers Squibb., M. Kihara: None declared, K. Amano Grant/research support from: Pfizer Japan Inc., R. Yoshimi Grant/research support from: Bristol-Myers K.K., M. Inoo: None declared, H. Dobashi: None declared, T. Sugihara Grant/research support from: Takeda Pharmaceutical Co. Ltd., Mitsubishi-Tanabe Pharma Co., Chugai Pharmaceutical Co., Ltd., Janssen Pharmaceutical K.K, Santen Pharmaceutical Co., Ltd., Astellas Pharma Inc., Bristol Myers Squibb K.K., Abbvie Japan Co., Ltd., Takeda Pharmaceutical Co., Ltd, and Astellas Pharma Inc., T. Hidaka: None declared, H. Kohsaka Grant/research support from: Ayumi Pharmaceutical Co., Pfizer Japan Inc., Mitsubishi-Tanabe Pharma Corp., Takeda Pharmaceutical Co. Ltd., AbbVie Japan Co. Ltd., Eisai Co., Ltd., Chugai Pharmaceutical Co. Ltd., Bristol-Meyers Squibb, Astellas Pharma Inc., UCB Japan, N. Miyasaka: None declared, M. Harigai Grant/research support from: Ayumi Pharmaceutical Co., Chugai Pharmaceutical Co., Ltd., Eisai Co., Ltd., Nippon Kayaku Co., Ltd., Taisho Toyama Pharmaceutical Co., Ltd., Takeda Pharmaceutical Co., Ltd., Mitsubishi Tanabe Pharma Co., Teijin Pharma Ltd., Eisai, Pfizer Japan Inc., Sanofi-Aventis KK., Santen Pharmaceutical Co., Ltd and Sekisui Medical Co., Ltd.

DOI: 10.1136/annrheumdis-2017-eular.3335

\section{THU0171 INCREASED FUNCTIONAL ACTIVITY OF FOXP3+REGULATORY T CELLS IN THE PERIPHERAL BLOOD OF DMARDS-NAÏVE METHOTREXATE-TREATED PATIENTS WITH EARLY RA USING}

A. Avdeeva $^{1}$, Y.P. Rubtsov ${ }^{2}$, T.V. Popkova ${ }^{1}$, D.T. Diykanov ${ }^{2}$, E.L. Nasonov ${ }^{1}$. ${ }^{1}$ Nasonova Research Institute of Rheumatology; ${ }^{2}$ Lomonosov Moscow State University, Moscow, Russian Federation

Objectives: To analyze the effect of methotrexate (MT) therapy on the percentage and absolute number of FoxP3+ regulatory T (Treg) cells in the peripheral blood of MT-naïve patients (pts) with early RA we used Treg immunostainingwith antibodies to surface markers and Foxp3 with subsequent flow cytometric analysis Methods: 45 MT-naïve pts with early RA (39 females, Me;lQR age 52.0 (32.5-57.5) years, RA duration 5 (4-6) months, DAS 285.01 (4.18-5.8), RF positive $-71.1 \%$, ACPA - positive- $88.9 \%$ ) were included into the study. All pts were administered subcutaneous MT as the first-line DMARD at $10 \mathrm{mg} /$ week with rapid dose escalation up to $20-25 \mathrm{mg} / \mathrm{week}$. Human blood mononuclear cells were isolated from whole venous blood by FicollHypaque centrifugation and subjected to multicolor flow cytometry analysis. Tregs were stained for different surface markers, and proportions of marker-positive subsets (FoxP3+CD25+; CD152+surface; CD152+intracellular; FoxP3+CD127-;
CD25+CD127-; FoxP3+ICOS+; FoxP3+CD154+; FoxP3+CD274+) were determined; 20 healthy donors made the control group

Results: After 24 weeks of MT therapy value DAS 28 was 3.1 (2.7-3.62); SDAI 7.4 (4.2-11.4); DAS 28 remission/low disease activity was achieved in $22(56.4 \%)$ pts, based on SDAI - in $25(64.1 \%)$ pts; while MT failure based on EULAR response was documented in $4(10.3 \%)$ pts. Lower percentages of FoxP3+CD25+ cells ((5.53 (4.09-6.48 vs 6.92 (5.84-7.96)), percentages and absolute number of FoxP3+ICOS+ cells ((6.91 (2.14-11.47) vs 10.83 (9.27$13.7) ; 0.0035$ (0.0013-0.0067) vs $0.0068(0.0039-0.009))$, and percentages and absolute number of FoxP3+CD154+ cells $((0.47(0.19-0.83)$ vs $1.51(1.12-2.08)$; $0.0002(0.00009-0.0005)$ vs $0.00087(0.00047-0.0014))$, and FoxP3+CD274+ T-cells $(0.63(0.34-1.49)$ vs $1.94(1.16-2.25) ; 0.0003(0.0002-0.00065)$ vs 0.001 (0.0006-0.0016), $p<0,05$ in all cases) were documented in early RA pts versus healthy donors.

An increase in the percentage of CD4+cells (from 45.0 (38.0-49.2) to 46.8 (39.953.2)); an increase in the relative and absolute number of CD152+surface $0.65(0.22-1.67)$ vs $2.07(1.11-3.81) ; 0.0002(0.0001-0.0008)$ vs 0.0007 (0.0004-0.002); and moderate decrease in the relative and absolute number of FoxP3+ICOS+ cells 5.3 (2.1-11.3) vs 4.1 (1.6-6.6); 0.002 (0.001-0.006) vs $0.0015(0.0006-0.003), p<0.05$ in all cases, was registered after 24 weeks of MT therapy. After 24 weeks of MT therapy the level of CD152+surface in the RA pts group was higher compared with healthy donors $2.1(1.11-3.81)$ and 0.51 $(0.34-1.2) ; 0.0007(0.0004-0.002)$ and $0.0003(0.00014-0.0008)$, respectively, $\mathrm{p}<0.05$

Treg levels and phenotype were analyzed in pts groups based on MT efficacy by 24th week of treatment. Higher percentages and absolute number of FoxP3+CD274+ cells (1.25 (0.43-2.3) $0.0004(0.0002-0.001)$ were detected in patients achieving SDAI remission $(n=25)$, compared to pts with moderate disease activity (SDAl >11 n=14) (0.44 (0.2-0.69) $0.00016(0.0001-0.0004), p<0.05)$

Conclusions: MT therapy in early RA pts results in increased Treg suppressor activity according to growing percentages and absolute number of CD152+surface and FoxP3+CD274+ cells, more pronounced among pts, achieving remission/low disease activity following treatment. Increased levels of these markers are indicative of improved Tregs functional activity after successful MT therapy

Disclosure of Interest: None declared

DOI: 10.1136/annrheumdis-2017-eular.3668

\section{THU0172 IMPROVEMENT OF DISEASE ACTIVITY IN A 5-YEAR COHORT OF RHEUMATOID ARTHRITIS PATIENTS TREATED UNDER TREAT TO TARGET RECOMMENDATIONS AND A MULTISPECIALITY CARE MODEL RECEIVING CONVENTIONAL DMARD THERAPY}

\section{P. Santos-Moreno $^{1}$, D. Gomez ${ }^{1}$, E. Castillo ${ }^{1}$, R. Giraldo ${ }^{1}$, G. Ballesteros ${ }^{1}$,} L. Villarreal ${ }^{2}$, J. Bello ${ }^{3}$, D. Buitrago-Garcia ${ }^{3} .{ }^{1}$ Rheumatology; ${ }^{2}$ Psychology and processes; ${ }^{3}$ Epidemiology, Biomab, Center for Rheumatoid Arthritis, Bogota, Bogota, Colombia

Background: Treat to Target (T2T) strategy becomes from the need to develop therapeutic targets and tools to achieve defined outcomes in rheumatoid arthritis $(\mathrm{RA})$, this strategy has become recognized as a standard of good practice embodying the principle that rapid attainment of remission, or low disease activity, can halt joint damage and maintain good quality of life.

Objectives: The aim of this study was to describe global change in Disease Activity Score 28 (DAS28) using T2T strategy for a 5 year period in patients with conventional DMARD therapy in a large cohort of patients from a Colombian specialized in RA center with multidisciplinary care model (MCM).

Methods: A descriptive dynamic cohort study was performed. Records of patients using conventional DMARD treatment from specialized in RA center were reviewed; those patients were followed-up under T2T standards. Clinical follow-up was according to DAS28 as follows: every $3-5$ weeks (DAS28 > 5.1), every $7-9$ weeks (DAS28 $\geq 3.1$ and $\leq 5.1$ ), and every $11-13$ weeks (DAS28 $<3.1$ ). Therapy had to be adjusted with DAS28 $>3.2$ unless patient's conditions don't permit it. MCM model means that every patient is seen by the other specialties involved in care as a minimum three times a year. We divided patients in three groups: low disease activity (LDA), moderate disease activity (MDA) and severe disease activity (SDA) patients. Descriptive epidemiology was done, percentages and averages were calculated; the median of each variable was analyzed using t-Student assuming normality for DAS28 distribution and the level activity disease was analyzed using Pearson's statistics.

Results: We included 1443 patients, $84 \%$ were women and $16 \%$ were men. Mean age was $62 \pm 11$ years; mean DAS28 at beginning was $4.0 \pm 1$; regarding disease activity $57 \%$ were in moderate disease activity and $17 \%$ in severe disease activity. While at 5 year follow up mean DAS was $2.92 \pm 0.65,48 \%$ achieved remission, $30 \%$ low disease activity, with decrease to $20 \%$ in moderate disease activity and $2 \%$ in severe disease activity.

\begin{tabular}{|c|c|c|c|c|}
\hline ACTIVITY LEVEL & TIME $0 \mathrm{n}(\%)$ & 2012-2013 & 2013-2014 n (\%) & 2014-2016 n (\%) \\
\hline REM & - & $380(26 \%)$ & \begin{tabular}{|l|}
$530(37 \%)$ \\
\end{tabular} & \begin{tabular}{|r|}
$698(48 \%)$ \\
\end{tabular} \\
\hline LDA & $380(26 \%)$ & $495(34 \%)$ & $487(34 \%)$ & $437(30 \%)$ \\
\hline MDA & $782(54 \%)$ & $439(30 \%)$ & $401(28 \%)$ & $284(20 \%)$ \\
\hline SDA & $281(19 \%)$ & $129(9 \%)$ & $25(2 \%)$ & $24(2$ \\
\hline
\end{tabular}

Conclusions: There is a significant improvement of DAS28 in a cohort of RA 